\title{
Pokémon Go - The Next Innovation in Mobile Gaming: Marketing Implications
}

\author{
Erwin Premchan ${ }^{1}$, Benjamin Wakeman ${ }^{1} \&$ Raj Devasagayam ${ }^{1}$ \\ ${ }^{1}$ Center for Undergraduate Research and Creative Activity, Siena College, Loudonville, New York, USA. \\ Correspondence: Raj Devasagayam, Professor of Marketing, Director, Center for Undergraduate Research and \\ Creative Activity, Siena College, 515 Loudon Road, Loudonville, New York 12211, USA.
}

Received: December 2, 2016

Accepted: February 20, 2017

Online Published: March 14, 2017

doi:10.20849/abr.v2i1.123

URL: https://doi.org/10.20849/abr.v2i1.123

\begin{abstract}
This research examines the past and present of innovative gaming. We explore the events and innovations that have led to the success of Pokémon Go and raise issues and questions that invite marketers to further delve into an agenda of research that would be truly valuable to practitioners and academicians alike.
\end{abstract}

Keywords: Pokémon Go, history, virtual gaming, augmented reality, marketing strategy, ethical issues

On July $6^{\text {th }}$ Pokémon Go was released into the world and it caught the attention of many like wildfire. The card game that a great majority of millennials grew up with had been transformed into a real life mobile game. With the first games to be released outside of Japan being, Pokémon Red version and Pokémon Blue version, to North America on September 28, 1998, Australia and New Zealand on October 23, 1998 and in Europe on June 10, 1999. Those who have played the game since the beginning have always dreamed that Pokémon would be real and they could live through the life of Ask Ketchum from the popular animated TV series Pokémon. Well now that they can, with Pokémon GO it has gained enormous amounts of popularity spreading too many people that didn't even know what Pokémon were until now. Looking back through the years no one would have thought that they would be able to go outside their house walk around a few blocks and catch a Pickachu. But here we are, in 2016, the era where anyone with a smartphone can become the very best there ever was... a Pokémon master. Nintendo had truly outdone themselves, according to investors.com, "U.S. shares of the Japanese video game Nintendo (NTYDOY) soared to their highest level in five years." The day of the game's release Nintendo stocks were at $\$ 17.63$ per share, the shares continued to soar and peaked at $\$ 30.25$; on July $22^{\text {nd }}$ Nintendo closed at $\$ 29.00$ per share. Though not surprising this has not been the first time Nintendo gaming has brought about a change in the gaming industry.

Nintendo had a hand to play in saving the gaming industry from the crash that took place in 1983. Jeff Ryan writes that due to the excessive release of bad video games and the presence of such a multitude of gaming platforms to play on caused the video game crash in 1983. This would lead many gaming companies to be put out of business. Cohen writes in the past Nintendo has revolutionized the industry even saved it from the video game crash in 1983 with the release of Super Mario Bros. Nintendo Entertainment System was the video game console that allowed the gaming industry to bounce back on its feet but without the release of a perfect game to go along with the console the industry would still be dead. Today, Mario is a video game character that is known far and wide. Nintendo has various spin offs of the original character and his little brother Luigi. A few of these video game spin offs are Super Smash Brothers, Mario Kart, Mario Party, Mario Tennis, Dr. Mario and much more.

Pac Man was another famous video game icon that came out of Nintendo. First starting out as an arcade game it is a video game that was present when the video game industry was being flooded with shooting and war games said Christine Champagne in her article "How "Pac-Man" changed games and culture." A simple 2 dimensional game that came out more than 30 years ago and is to this day still one of the most well known and most played games in history. Pac Man's lead designer Iwatani Tohru wanted his game to have a different feel to it. Henry Lowood writes Tohru wanted to create a game that had less violence and would appeal to a female audience as well as a male audience. Over the years we have seen Pac man being transformed from just a simple arcade game into a piece of culture says Lowood. For example, the song Pac man fevers by Buckner and Garcia, which hit the top 40 songs when it came out in 2002. These two ideas came out of Nintendo and are recognized 
worldwide; today, fans have developed mobile apps to play either Pac man or Mario through smartphones, there are even websites dedicated to these two characters and the multitude of games that came with their creations. Now Pokémon Go is here to give us a new wave of change that is augmented reality gaming.

When we look at the impact Pokémon Go has had on the community since its release it is fair to say that similar to Super Mario Bros. and Pac Man, Pokémon Go is a new wave that will change the way gamers, and game designers view gaming. Video gamers are often viewed as introverts that spend their days slaving away at playing a mindless video game and having no social interaction whatsoever. However, Pokémon Go has broken that standard and now has its users doing a multitude of things that would not make sense by the traditional definition of gamers. Users are getting physical exercise, socializing with other users and are even getting more familiar with the history and art work in their surroundings. The Knowledge@Wharton article, "How Pokémon Go took augmented reality mainstream." Shows insight on how Pokémon Go has started the new gaming trend. Kartir Hosanagar, professor of operations at Wharton says that Pokémon Go is one of the first games to bring together the digital world and the real world for a completely new experience.

Augmented reality has now officially been brought to the table that is mobile gaming; other games such as Ingress have also helped with the building up to the release of Pokémon Go. Where the augmented gaming industry goes from here is up to the big corporations and gaming companies, we might have to wait a while for the next wave of game changers to hit the industry. The app is easy to play and easy to get attached too, but there is one issue that should give pause to users of the app -- "User Agreements." Are gamers even aware of this agreement? Do users know what they are agreeing too?

The game uses augmented reality (AR), which is new in the gaming industry. To use AR in this game it prompts the player when they first open the app to register with the Pokémon training club or with a Gmail account to play the game. After this it asks for permission to use your information from your account and your location. If the player declines they are sent back to the opening screen and can't play the game.

Agreeing to the user agreement allows the app permission to access, collect and use information on from the third party services used to play the game (such as Google accounts). This grants the app to use information from the third party services to be shared to identify the user. One could reasonably argue that most users don't understand the full extent of these user agreements and how much privacy one is giving up.

Nobody likes the feeling of being watched, yet user agreements seek and obtain permission to do so. When consumers agree to the privacy policies of apps and services such as Pokémon GO they rarely read what terms they are agreeing too. A basic assumption would be that only a user's location is shared and nothing else, but they would be wrong. Much more is shared when Pokémon GO is played. After closely examining the private policy of Pokémon GO there could be a number of issues that could cause concern. A brief description of these would be allowing Pokémon GO access to your Gmail accounts (or other third party service), your mobile device itself, and the right for Pokémon GO as well as third party services to use information collected as a "business asset."

This can be disturbing to many people, but the user agreement does state that company has access to "non-identifying information." Basically saying that the app has access to all of the things stated above, but the information gathered won't identify a user specifically. The information gathered is statistical and can be used to see what trends are happening with users of the app. While the game promises anonymity of users specifically, it does have the raw material to easily could do so.

As one reads in more detail one could discern that private information has already been collected by companies such as, Google for a long time. It is just that with the popularity and excessive use of Pokémon GO this topic behooves a closer scrutiny. Google is power house in the world of the internet today. Google has access to so much information from so many people around the world. Google is connected to maps, YouTube account, and anything that uses a Gmail account to log into just to name a few. With so much data being shared constantly around the world Google has access to an insurmountable amount of information which could be used to identify a specific person if needed. By quickly agreeing to these apps and services consumers might be giving away more and more of their privacy on the internet. The legality for these apps and services to have access to all of this information as long as we simply agree to the user agreements may raise ethical concerns, all the same.

The short answer to legality seems - yes. It is completely legal for Pokémon GO to have access to user information per the terms and conditions of the User Agreement. There are privacy laws in place that state that "legal responsibility to take steps to properly secure or dispose" of any information used by that company. If Pokémon GO didn't abide by these laws it wouldn't be allowed in the United States and it states in the privacy policy of Pokémon GO that "following termination... of your Account, Niantic, its clients, affiliates, or service 
providers may retain information and user content for a commercially reasonable time period for backup." In layman's terms, Pokémon GO holds onto user information after consumers terminate their account for a certain amount of time, before disposing of it at the company's discretion.

However, there may be ways to keep information more private if users are uncomfortable with the amount of access Pokémon Go has when using the app. One way is to simply not play the game, however if someone must play and wants to keep most of their information private they might need to create a second email account. By doing this Pokémon GO would have access to an account not used for personal information. The catch is that the app will still have access to user's exact location, but if users can live with that then they will be able to enjoy the game.

Despite Pokémon GO's overall success, there are indications that the game is losing momentum in the US. Some of the reasons attributed for this decline in the market are as follows. First, there are bugs in the game, as expected from something this big, with game crashes, and server issues. As the game moves forward server issues and game crashes need fixing. In effect, the game is a victim of its own popularity. In this age of fleeting attention spans, if users can't play the game because it doesn't perform properly for technical reasons, gamers lose interest and move on. Secondly, the game play mechanics are extremely different than the original games that gamers are used to. The original games were turn based and battles could be fought anywhere in the game. In the app version battles can only happen at "gyms" and they are not turn based. This can turn classic Pokémon fans away. The creators assure us that later in the game, players will be able to battle against each other if they are in physical proximity. Finally, the new consumer behavior models that are emerging necessitate educating the consumers to innovative advances.

In conclusion, Pokémon GO is a revolutionary game that has got everybody talking about it. Many concerns with the game revolve around its privacy policy, diffusion of innovation, consumer learning, technological issues, and ethical issues. There is absolutely no indication that Pokémon GO intends to use information collected to negatively affect their customers. The preceding narrative is a call for marketers to launch a new stream of research in light of a truly innovative product with a phenomenal global following. We hope that our analyses will spark a seminal stream of research that matches the creative genius of this innovative product. Meanwhile, happy hunting!!

\section{References}

Champagne, C. (2013). How "Pac-Man" Changed Games and Culture. Web, 18 July 2016.

Cohen, D.S. (2016). Super Mario Bros. The Game That Saved Video Games. Super Mario Bros. The Game That Saved Video Games, Web.

How Pokémon Go Took Augmented Reality Mainstream. Knowledge@Wharton How Pokémon Go Took Augmented Reality Mainstream Comments. Web, 21 July 2016.

Lowood, H.E. (2016). Pac-Man. Pac-Man. Encyclopedia Britannica. Web, 16 July 2016.

Ryan, J. (2011). Super Mario: How Nintendo Conquered America. New York: Portfolio Penguin.

Seitz, P. (2016). Nintendo Stock Soars on Hit Mobile Game 'Pokémon Go'. Investor's Business Daily, 11 July 2016. Web, 25 July 2016.

Wolf, M.J.P. (2012). Before the Crash: Early Video Game History. Detroit: Wayne State UP.

\section{Copyrights}

Copyright for this article is retained by the author(s), with first publication rights granted to the journal.

This is an open-access article distributed under the terms and conditions of the Creative Commons Attribution license (http://creativecommons.org/licenses/by/4.0/). 